\title{
Autoconhecimento como fator importante no diagnóstico de câncer de mama: estudo de caso
}

\author{
Self-knowledge as an important factor in breast cancer diagnosis: a case study
}

\section{Autoconocimiento como factor importante en el diagnóstico de cáncer de mama: estudio de caso}

Recebido: 12/08/2019

Aprovado: 21/12/2019

Publicado: 17/02/2020
Heloísa Silva Guerra ${ }^{1}$ Oemis Eduardo Xavier ${ }^{2}$ Ruth da Silva Rêgo ${ }^{3}$ Jorge Henrique Assunção Dias ${ }^{4}$ Gustavo da Paixão Alcântara ${ }^{5}$

Trata-se de um estudo de caso, cujo objetivo foi relatar a dificuldade na detecção de uma neoplasia mamária, em que o diagnóstico orientou-se por um único achado clínico. 0 período considerando das informações foi desde 2016, mas realizado no primeiro semestre de 2019, em cidade do interior de Goiás. Houve subdiagnóstico por vários profissionais de saúde, após três mamografias e duas ultrassonografias sugestivas de benignidade. A despeito dos resultados, a paciente buscou hospital de referência e a repetição de exames mostraram ainda benignidade, mas a adaptação de aparelhos no exame mostrou malignidade, a ponto de que a paciente chegou a fazer quimioterapia e depois mastectomia. Exercitar o autoconhecimento é ferramenta importante que contribui para emancipação feminina e expressão de seu empoderamento, bem como, a anamnese completa, a escuta qualificada e o exame físico detalhado, são essenciais para o diagnóstico preciso; e, tratamento oportuno com vistas à melhoria da qualidade de vida da paciente.

Descritores: Neoplasias da mama; Diagnóstico; Mamografia.

It is a case study whose objective was to report the difficulty in detecting a breast cancer, in which the diagnosis was guided by a single clinical finding. The period considering the information was from2016, but held in the first half of 2019 in the city of Goiás. There was underdiagnosis by various health professionals, after three mammograms and two ultrasound suggestive of benignity. Despite the results, the patient sought a referral hospital and tests repetition also showed benignity, but the adaptation of devices in the exam showed malignancy, to the point that the patient underwent chemotherapy, then mastectomy. Exercising self-knowledge is an important tool that contributes to female emancipation and expression of her empowerment, as well as the complete history, qualified listening and detailed physical examination are essential for accurate diagnosis; and timely treatment with a view to improving the quality of life of the patient.

Descriptors: Breast neoplasms; Diagnosis; Mammography.

Se trata de un estudio de caso, cuyo objetivo fue reportar la dificultad en la detección de una neoplasia mamaria, en la que el diagnóstico se orientó por un único hallazgo clínico. El período considerado de la información fue desde 2016, pero realizado en el primer semestre de 2019, en ciudad del interior de Goiás. Hubo subdiagnóstico por varios profesionales de salud, después de tres mamografías y dos ecografías sugestivas de benignidad. A pesar de los resultados, la paciente buscó hospital de referencia y la repetición de exámenes mostraron aún benignidad, pero la adaptación de aparatos en el examen mostró malignidad, hasta el punto de que la paciente llegó a recibir quimioterapia y después mastectomía. Ejercitar el autoconocimiento es una herramienta importante que contribuye a la emancipación femenina y expresión de su empoderamiento, así como, la anamnesis completa, la escucha cualificada y el examen físico detallado, son esenciales para el diagnóstico preciso; y, tratamiento oportuno con vistas a la mejora de la calidad de vida de la paciente.

Descriptores: Neoplasias de la mama; Diagnóstico; Mamografía.

1. Fisioterapeuta. Especialista em Fisioterapia Traumato-ortopédica. Especialista em Terapêutica do Movimento Humano. Especialista em Saúde do Trabalhador. Mestre em Saúde Coletiva. Doutoranda em Saúde Coletiva pela Universidade do Vale do Rio dos Sinos. Professora Adjunta I da Faculdade de Medicina da Universidade de Rio Verde (UniRV), Campus Aparecida de Goiânia, Goiás, Brasil. ORCID: 0000-0002-0617-8112 E-mail: heloisasguerra@gmail.com

2. Acadêmico do curso de graduação em Medicina pela UniRV, Campus Aparecida de Goiânia, Goiás, Brasil. ORCID: 0000-0001-9852-5312 E-mail: oemiseduardo@gmail.com

3. Acadêmica do curso de graduação em Medicina pela UniRV, Campus Aparecida de Goiânia, Goiás, Brasil. ORCID: 0000-0002-4729-6465 E-mail: ruthrego29@gmail.com

4. Acadêmico do curso de graduação em Medicina pela UniRV, Campus Aparecida de Goiânia, Goiás, Brasil. ORCID: 0000-0001-6446-2552 E-mail: jorgehadias1@gmail.com

5. Acadêmico do curso de graduação em Medicina pela UniRV, Campus Aparecida de Goiânia, Goiás, Brasil. ORCID: 0000-0002-1058-6207 E-mail: gustavopaixaoalcantara@hotmail.com 


\section{INTRODUÇÃO}

$\mathbf{0}$ câncer de mama (CM), excluindo o câncer de pele não melanoma, é o câncer mais incidente no mundo e no Brasil, exceto na região norte ${ }^{1}$. 0 CM é raro antes dos 35 anos e sua incidência cresce exponencialmente com a idade ${ }^{2}$.

Dentre os fatores de risco destaca-se idade maior que 50 anos, menarca antes dos 12 anos, menopausa após 55 anos, primeira gravidez após 30 anos, nuliparidade, uso de anticoncepcional hormonal ou terapia de reposição hormonal, etilismo, tabagismo, sobrepeso, exposição à radiação ionizante, história familiar de $\mathrm{CM}$ ou câncer de ovário e mutações nos genes BRCA 1 e BRCA $2^{3}$.

A prevenção se divide em primária e secundária. A prevenção primária está relacionada a controle de fatores de risco e, em mulheres BRCA 1 e 2 positivos, mastectomia profilática ou uso de tamoxifeno. A prevenção secundária está relacionada ao rastreio via mamografia (MMG) bianual para mulheres de 50 a 69 anos $^{3}$, sendo que a ultrassonografia (USG) de mamas e a ressonância nuclear magnética (RNM) podem ser indicadas em casos específicos ${ }^{4}$.

As manifestações clínicas mais comuns do CM são: lesão eczematosa da pele que não responde a tratamentos tópicos, retração na pele da mama, mudança no formato do mamilo, aumento progressivo da mama e, sinais de edema, nódulo mamário em mulheres com mais de 30 a 50 anos, que persistem por mais de um ciclo menstrual, nódulo mamário de consistência endurecida e fixo ou que vem aumentando de tamanho em mulheres adultas de qualquer idade $^{5}$.

O diagnóstico se dá pelo exame clínico geral, exames de imagens e anatomopatológico (AP). Dentre os exames de imagem, a MMG é a mais utilizada por sua acessibilidade e baixo custo, porém apresenta limitações como a diferenciação entre estruturas císticas e sólidas, avaliação de mamas densas ou que apresentem alterações fibrocicatriciais, que podem ser resolvidas com associação à USG ou RNM 6 .

A MMG é feita com a mulher colocada de pé em frente ao mamógrafo, com as mamas descobertas sem o uso de cremes hidratantes, perfumes e desodorantes que possam gerar artefatos. Em seguida se posiciona as mamas sobre o aparelho, seguida de uma compressão entre duas placas planas para a realização das imagens. A duração total do exame se dá em poucos minutos ${ }^{7}$.

Os fatores prognóstico do CM inclui o grau de diferenciação celular, o estadiamento e o exame imunohistoquímico, que avalia a expressão anormal do receptor HER2, associado a maior agressividade da doença, e ausência de receptores hormonais ${ }^{5,8}$. 0 tratamento do CM deve ser individualizado e pode incluir quimioterapia, radioterapia, mastectomia parcial ou total, linfadenectomia, terapia hormonal e anticorpo monoclonal ${ }^{9}$. Diante disso, o objetivo deste estudo foi relatar a dificuldade na detecção de uma neoplasia mamária, em que o diagnóstico orientou-se por um único achado clínico.

\section{MÉTODO}

Trata-se de um estudo descritivo do tipo estudo de caso, realizado por meio do acompanhamento de uma paciente com câncer de mama não identificado mediante exames protocolares normais.

O trabalho de campo envolveu a coleta dos dados em uma Unidade Básica de Saúde (UBS) no município de Cabeceiras - GO, a partir dos registros de fichas, prontuário e exames, no período de maio a julho de 2019.

O estudo foi aprovado pelo Comitê de Ética em Pesquisa da Fundação do Ensino Superior de Rio Verde - FESURV - Universidade de Rio Verde por meio do Parecer no 3.193.259, CAAE 07803319.8.0000.5077; e foi assegurado à participante que sua cooperação era livre, consentida, esclarecida e que o aceite seria dado mediante assinatura do Termo de Consentimento Livre e Esclarecido (TCLE). 


\section{RESULTADOS}

Paciente LFC, 44 anos, casada, professora, natural e procedente de Cabeceiras-GO. Relata menarca aos 14 anos G2 P2(n+c) A0, primeiro filho aos 31 anos e segundo aos 35, tendo amamentado ambos os filhos por 2 anos, uso de anticoncepcional oral combinado desde os 22 anos de idade, sendo que no período de lactação do primeiro filho usou minipílula e após o segundo parto realizou laqueadura.

Há cerca de 2 anos, aos seus 42 anos de idade, percebeu uma depressão no mamilo direito, sem nódulo palpável, dor e descarga papilar. Procurou o médico da UBS de seu município que solicitou uma mamografia (MMG). No retorno, em setembro de 2016, a MMG trazia um achado BIRADS 2 (achados benignos) e o profissional a orientou que possivelmente seria leite retido.

Não satisfeita com a conduta do médico, a paciente relata que procurou um mastologista em outra cidade, o qual solicitou MMG e ultrassonografia das mamas (USGM). No retorno, a MMG (que foi realizada em clínica diferente que a primeira) trazia um resultado BIRADS 3 (achados provavelmente benignos) e a USGM dentro das normalidades. Diante desses achados, o mastologista concordou com a conduta inicial do médico da UBS.

Com passar do tempo, a paciente percebeu que a retração mamilar estava se intensificando e resolveu procurar outro médico da sua cidade em janeiro de 2017, que ao examiná-la e tomar nota de sua história clínica, a encaminhou para um serviço de referência hospitalar. Ao ser atendida neste serviço, em posse de nova MMG constando BIRADS 3, o médico relatou que as alterações não eram dignas de preocupação, mas ao examiná-la percebeu que os achados de imagem não estavam compatíveis com a clínica. Em seguida, a paciente foi levada ao mamógrafo, e ao realizar outra MMG, não encontrou achados sugestivos de malignidade. Diante disso, o médico se reuniu com a equipe e decidiram mudar o compressor. Ao realizar nova MMG, foram encontradas alterações sugestivas de malignidade (BIRADS 5).

A equipe médica solicitou uma biópsia de fragmento com agulha (BFA) ou core biopsy, que evidenciou carcinoma mamário infiltrante lobular grau I; além disto pediu também: Cintilografia, Tomografia de tórax, USG pélvica e de abdome, Citologia oncoparasitária, Hemograma, AST, ALT; os quais tiveram resultados dentro da normalidade, além do imunohistoquímico.

No retorno, já em posse dos exames citados, a equipe prescreveu tratamento quimioterápico com intenção neoadjuvante. A paciente realizou oito ciclos de quimioterapia com intervalo de 21 dias, sendo os quatro primeiros ciclos de Adriamicina e Ciclofosfamida e os quatro últimos de Paclitaxel, com término em 1ํo de março de 2018.

Semanas após, a paciente teve pneumonia, sendo internada para realização da antibioticoterapia no mesmo hospital de referência.

Após resolução do quadro infeccioso, foi marcada a mastectomia, realizada no dia 7 de abril de 2018. Atualmente a paciente apresenta-se em bom estado geral, sem queixas urinárias e intestinais, exame dos aparelhos cardiovascular e respiratório sem alterações, queixa de amenorreia e dor em choque nas mamas que não cessa com analgésicos ou opioides desde o início da quimioterapia. Paciente nega antecedentes de câncer na família e permanece em acompanhamento no serviço de referência oncológica.

\section{DISCUSSÃO}

Considerando o caso em questão, percebeu-se a importância do autoconhecimento corporal e da contestação, por parte da paciente, de um resultado não condizente com sua condição clínica. Mesmo com três resultados mamográficos negativos para malignidade, a paciente insistiu na busca pela assistência médica, pois acreditava que algo não estava dentro da normalidade, reforçando sua autonomia e autocuidado. A autonomia pressupõe liberdade de ação e pensamento, permitindo que a pessoa aja livremente para escolher as opções que lhe são apresentadas. 
Estudo realizado com 400 mulheres, demonstrou que a maioria significativa delas não praticava o autoexame das mamas mensalmente, e que a cada dez mulheres, apnas seis realizavam a mamografia a cada dois anos ${ }^{10}$. Embora não seja recomendado pelo Instituto Nacional do Câncer (INCA) como forma de rastreamento, o autoexame é um método específico e sistemático para detecção de alterações nas mamas, além de ajudar no autoconhecimento do próprio corpo, auxiliando na identificação precoce de alterações mamárias, visto que as mulheres podem se dirigir a um serviço motivadas por esses achados, evitando danos futuros ${ }^{10}$.

No modelo atual do sistema de saúde, e na perspectiva da Saúde Coletiva, a educação em saúde é essencial na conscientização crítica dos indivíduos, envolvendo-os no processo de tomada de decisões relacionadas às melhorias de suas condições de saúde e o meio em que vivem $^{11}$.

As diretrizes para detecção precoce do câncer de mama no Brasil recomendam o rastreamento com mamografia em mulheres a partir dos 50 anos de idade ${ }^{12}$. Esse rastreamento é adiantado para mulheres de alto risco para o $\mathrm{CM}$, como as que possuem mutações no BRCA 1 e BRCA 2, histórico de CM em parentes de primeiro grau e mulheres que realizaram radioterapia no tórax. A paciente em questão não apresentava nenhum dos fatores de alto risco, assim, pelas diretrizes do Ministério da Saúde (MS), ela não se enquadraria na população coberta pelo rastreamento. Por outro lado, a Federação Brasileira das Associações de Ginecologia e Obstetrícia (FEBRASG0), indica o rastreamento anual para todas as mulheres acima de 40 anos de idade, e nesse caso a paciente se enquadraria ${ }^{13}$.

A abordagem metodológica para a detecção precoce do câncer de mama, inclui, além do rastreamento, estratégias para diagnóstico precoce nos casos com sinais e sintomas suspeitos. Porém, muitos são os desafios enfrentados para a implementação das diretrizes, como o fato de todos os procedimentos necessários não serem ofertados em um único serviço; o aumento da demanda associada ao rastreamento mamográfico e a não adesão dos profissionais às diretrizes ministeriais ${ }^{12}$.

Outro ponto importante e controverso a ser considerado é a questão do sobrerrastreamento, que ocorre no Brasil, muito em função do rastreamento mamográfico ser oportunístico, fazendo com que a periodicidade de realização do exame se afaste da recomendada. Uma coorte com 13.387 mulheres apontou que o sobrerrastreio atingiu 21\% delas, tendo a maioria dos casos ocorrido após 1 ano de mamografia normal ${ }^{14}$. Essa situação onera o sistema de saúde, uma vez que direciona uma quantia significativa de recursos para a realização de exames desnecessários, que poderiam estar cobrindo as necessidades de mulheres que realmente necessitem do exame.

No presente estudo, onde três resultados de MMG não apontaram malignidade, o exame não se mostrou muito efetivo a princípio. Vários fatores podem interferir na sensibilidade da MMG, como: erro de posicionamento da paciente, baixa qualidade da mamografia, erro na interpretação do exame, mamas densas, tamanho da lesão mamária e lesão com características radiológicas equivalentes às do tecido normal ${ }^{15}$.

No caso em questão, foi possível observar que a dificuldade do diagnóstico é multifatorial, englobando tanto o foco de incidência ineficaz, que somente houve êxito ao alterar uma peça do mamógrafo para se adequar a mama da paciente e assim obter a incidência ideal para identificar a lesão; quanto o pequeno tamanho da lesão e sua semelhança com o tecido mamário normal, sendo percebido apenas por meio da core biopsy realizada posteriormente, que identificou ausência de microcalcificações, além de baixo pleomorfismo nuclear, presença de raras mitoses, ausência de necroses, moderada fibrose, leve infiltrado de mononucleares e invasão peritumoral não vista, demonstrando também ser uma lesão não extensa de componente in situ ${ }^{3,6}$.

As limitações da MMG citada sugerem que a simples avaliação de um exame de imagem não é suficiente para rastrear possíveis lesões malignas, ressaltando-se a relevância de uma anamnese completa e realização de exame físico detalhado. 
As principais vias de disseminação do CM são vasos linfáticos e via hematogênica e os principais sítios afetados por metástases são ossos, pulmões, pleura, fígado, medula óssea e ovários. Isso explica a necessidade de realizar Cintilografia óssea, tomografia de tórax, USG pélvica e de abdome, citologia oncoparasitária, hemograma, AST e ALT, que no caso da paciente não apresentaram alterações, afastando a possibilidade de doença metastática9 .

Estudo realizado com pacientes portadoras de câncer de mama na região Sul do Brasil demostrou que a maior parte dos carcinomas $(53,9 \%)$ apresentavam tamanho inferior a $2 \mathrm{~cm}$, sem comprometimento de linfonodos (57\%) e sem metástases à distância $(93,4 \%)^{16}$; o que reforça as características do caso em questão.

Em relação aos fatores de risco para câncer de mama, o caso aqui apresentado mostrou a idade da primeira gestação acima de 30 anos e uso de anticoncepcional oral combinado (que possui relação com o estímulo estrogênico) e, que leva ao aumento do risco de desenvolver câncer de mama quanto maior for o tempo de exposição ${ }^{3}$.

0 tratamento do CM visa o controle locorregional e sistêmico da doença, de forma a proporcionar uma boa qualidade de vida às pacientes e diminuir as taxas de mortalidade. A quimioterapia neoadjuvante é aquela realizada anteriormente ao procedimento cirúrgico, propondo diminuir o tamanho tumoral, evitar cirurgias mutiladoras e diminuir metástases a distância. Os quimioterápicos que normalmente são utilizados na QT neoadjuvante são as antraciclinas, ciclofosfamida, fluorouracil e o paclitaxel ${ }^{9,17}$, como no presente caso.

O carcinoma lobular invasor clássico, também conhecido como carcinoma lobular invasor grau I, tende a afetar mulheres mais jovens do que as variantes desse tipo histológico, cuja idade média de ocorrência é 57 anos ${ }^{18}$. A informação corrobora com o aparecimento precoce dessa neoplasia no caso em análise. A frequência de carcinoma lobular invasor varia entre 2 a 15\% dos CM e a sua característica histológica mais marcante é o padrão de disposição em fila indiana das células neoplásicas.

0 diagnóstico precoce visa contribuir para melhores opções de tratamento, bem como melhor prognóstico da doença. Com o avanço tecnológico e maiores possibilidades de avaliação, as cirurgias conservadoras têm ganhado mais espaço, em detrimento à realização das mastectomias; proporcionando tratamentos cirúrgicos menos invasivos e uma menor morbidade ${ }^{19}$.

\section{CONCLUSÃO}

O caso clínico apresentado chama a atenção para importância do autoconhecimento e autonomia da paciente no processo de produção da saúde; bem como do debate clínico para melhor abordagem do caso. A conscientização dos indivíduos e o estímulo ao autocuidado são fundamentais para o exercício dessa liberdade, além da divisão de responsabilidades entre profissionais e pacientes.

Exercitar o autoconhecimento é ferramenta importante que contribui para emancipação feminina e expressão de seu empoderamento, enquanto capacidade de realizar por si mesma as mudanças necessárias para sua evolução, não permitindo manipulações de qualquer natureza.

A anamnese completa, a escuta qualificada e o exame físico detalhado, são essenciais para o diagnóstico preciso; reforçando que a humanização e a investigação minuciosa colaboram para um cuidado mais efetivo e tratamento oportuno com vistas à melhoria da qualidade de vida das pacientes.

0 estudo realizado apresenta limitações, visto que, por se tratar de um caso único, suas conclusões não apresentam base para generalização. No entanto, em função do impacto negativo e as sérias consequências que podem ocorrer em virtude de um diagnóstico não realizado, torna-se relevante a divulgação de seus achados. 


\section{REFERÊNCIAS}

1. Instituto Nacional de Câncer José Alencar Gomes da Silva. Conceito e magnitude do câncer de mama [Internet]. Brasília, DF: INCA; 2019 [citado em 02 maio 2019]. Disponível em: https://www.inca.gov.br/controle-do-cancer-de-mama/conceito-e-magnitude

2. Chala LF, Barros N. Avaliação das mamas com métodos de imagem. Radiol Bras. [Internet]. 2007 [citado em 02 maio 2019]; 40(1):4-6. Disponível em: http://www.scielo.br/pdf/rb/v40n1/001.pdf

3. Facina T. Resenha. Diretrizes para a detecção precoce do câncer de mama no Brasil. Instituto Nacional de Câncer José Alencar Gomes da Silva. Rev Bras Cancerol. [Internet]. 2016 [citado em 02 maio 2019]; 62(1):59-60. Disponível em: http://www1.inca.gov.br/rbc/n_62/v01/pdf/10resenha-diretrizes-para-a-deteccao-precoce-do-cancer-de-mama-no-brasil.pdf

4. National Comprehensive Cancer Network. Clinical Practice Guidelines in Oncology [Internet]; 2017 [citado em 25 out 2018]. Disponível em: https://www.nccn.org/clinicalpracticeguidelinesinoncology2017.pdf

5. Brasileiro FG. Bogliolo: patologia. 8ed. Rio de Janeiro: Guanabara Koogan; 2011. 1524 p.

6. Bernardes NB, Sá ACF, Facioli LS, Ferreira ML, Sá OR, Costa RM. Câncer de mama X diagnóstico. Id On Line Rev Mult Psic. [Internet]. 2019 [citado em 02 maio 2019]; 13(44):87785. Disponível em: https://idonline.emnuvens.com.br/id/issue/view/61/showToc

7. Félix J, Félix J, Cássia M, Alves T, Brito T, Soares WDB. Mamografia: aspectos gerais. Rev Cient Multidiscipl Núcleo Conhec. [Internet]; 2017 [citado em 22 out 2018]. 2(13):447-54. Disponível em: https://www.nucleodoconhecimento.com.br/saude/mamografia-aspectosgerais?pdf $=6521$

8. Castralli HA, Bayer VML. Câncer de mama com etiologia genética de mutaçao em BRCA1 e BRCA2: uma síntese da literature. Braz J Health Rev. [Internet]. 2019 [citado em 02 maio 2019]; 2(3):2215-24. Disponível em: http://www.brjd.com.br/index.php/BJHR/issue/view/49

9. Hoff PMG. Tratado de oncologia. São Paulo: Atheneu; 2013. 2829 p.

10. Silva RP, Gigante DP, Amorim MHC, Leite FMC. Fatores associados à realização de mamografia em usuárias da atenção primária à saúde em Vitória, Espírito Santo. Epidemiol Serv Saúde [Internet]. 2019 [citado em 02 maio 2019]; 29(1):e2018048. Disponível em: http://www.scielo.br/pdf/ress/v28n1/2237-9622-ress-28-01-e2018048.pdf

11. Ribeiro KG, Andrade LOM, Aguiar JB, Moreira AEMM, Frota AC. Educação e saúde em uma região em situação de vulnerabilidade social: avanços de desafios para as políticas públicas. Interface (Botucatu, Online) [Internet]. 2018 [citado em 02 maio 2019]; 22(Supl 1):1387-98. Disponível em: http://www.scielo.br/pdf/icse/v22s1/1807-5762-icse-1807576220170419.pdf

12. Migowski A, Silva GA, Dias MBK, Diz MDPE, Sant'Ana DR, Nadanovsky P. Guidelines for early detection of breast cancer in Brazil. II - New national recommendations, main evidence, and controversies. Cad Saúde Pública [Internet]. 2018 [citado em 02 maio 2019]; 34(6):e00074817. Disponível em: http://www.scielo.br/pdf/csp/v34n6/en_1678-4464-csp34-06-e00074817.pdf

13. Urban LABD, Chala LF, Bauab SP, Schaefer MB, Santos RP, Maranhão NMA, et al. Recomendações do Colégio Brasileiro de Radiologia e Diagnóstico por Imagem, da Sociedade Brasileira de Mastologia e da Federação Brasileira das Associações de Ginecologia e Obstetrícia para o rastreamento do câncer de mama. Radiol Bras. [Internet]. 2017 [citado em 02 maio 2019]; 50(4):244-9. Disponível em: http://www.scielo.br/pdf/rb/v50n4/pt_0100-3984-rb50-04-0244.pdf

14. Rodrigues TB, Stavola B, Bustamante-Teixeira MT, Guerra MR, Nogueira AC, Fayer VA, et al. Sobrerrastreio mamográfico: avaliação a partir de bases identificadas do Sistema de Informação do Câncer de Mama (SISMAMA). Cad Saúde Pública [Internet]. 2019 [citado em 02 maio 2019]; 35(1):e00049718. Disponível em: http://www.scielo.br/pdf/csp/v35n1/16784464-csp-35-01-e00049718.pdf 
15. Sabino SMPS. A importância da qualidade dos exames de imagem em mama. Rio de Janeiro: Sociedade Brasileira de Mastologia; 2017 [citado em 15 abr 2019]. Disponível em: http://www.sbmastologia.com.br/medicos/wp-content/uploads/2017/09/A-

importa\%CC\%82ncia-da-qualidade-dos-exames-de-imagem-em-mama.pdf

16. Laila HJEA, Zenkner JRG, Araújo MC, Becker JDL, Pereira AD. Characterization of prognostic factors of breast cancer among women with condition attended by the Brazilian Unified Health System in the municipality of Bagé, Rio Grande do Sul, Brazil. Mastology [Internet]. 2019 [citado em 02 maio 2019]; 29(2):64-70. Disponível em: http://www.mastology.org/wpcontent/uploads/2019/08/MAS-v29n2_64-70.pdf

17. Costa MADL, Chagas SRP. Quimioterapia neoadjuvante no câncer de mama operável: revisão da literatura. Rev Bras Cancerol. [Internet] 2013 [citado em 02 maio 2019]; 59(2):261-9. Disponível em: https://rbc.inca.gov.br/site/arquivos/n_59/v02/pdf/14-quimioterapianeoadjuvante-no-cancer-de-mama-operavel-revisao-da-literatura.pdf

18. Oliveira CF, coordenador. Manual de ginecologia [Internet]. Lisboa: Permanyer Portugal; 2011 [citado em 10 jun 2018]. v. 2. Disponível em: http://www.fspog.com/fotos/editor2/portadillas_vol_ii.pdf

19. Correa JL, Oenning TA, Martins JHAF, Souza ME, Barbosa JPP, Dória MT, et al. Trends in breast cancer surgery in private patients under opportunistic screening. Mastology [Internet]. 2019 [citado em 02 maio 2019]; 29(2):79-85. Disponível em: http://www.mastology.org/wpcontent/uploads/2019/08/MAS-v29n2_79-85.pdf

\section{CONTRIBUIÇÕES}

Todos os autores tiveram iguais contribuições da elaboração, redação e revisão do estudo.

\section{Como citar este artigo (Vancouver)}

Guerra HS, Xavier OE, Rêgo RS, Dias JHA, Alcântara GP. Autoconhecimento como fator importante no diagnóstico de câncer de mama: estudo de caso. REFACS [Internet]. 2020 [citado em inserir dia, mês e ano de acesso]; 8(1):155-161. Disponível em: inserir link de acesso. DOI: inserir link do DOI.

\section{Como citar este artigo (ABNT)}

GUERRA, H. S.; XAVIER, O. E.; RÊGO, R. S.; DIAS, J. H. A.; ALCÂNTARA, G. P. Autoconhecimento como fator importante no diagnóstico de câncer de mama: estudo de caso. REFACS, Uberaba, MG, v. 8, n. 1, p. 155-161, 2020. Disponível em: inserir link de acesso. Acesso em: inserir dia, mês e ano de acesso. DOI: inserir link do DOI.

\section{Como citar este artigo (APA)}

Guerra, H.S., Xavier, O.E., Rêgo, R.S., Dias, J.H.A. \& Alcântara, G.P. (2020). Autoconhecimento como fator importante no diagnóstico de câncer de mama: estudo de caso. REFACS, 8(1), 155-161. Recuperado em: inserir dia, mês e ano de acesso de inserir link de acesso. DOI: inserir link do DOI. 